$12-17-2020$

\title{
Intrahistiocytic Storage of Clofazimine Crystals in a Cat
}

\author{
Nathan D. Helgert \\ Murray State University, nhelgert@murraystate.edu \\ Debra L. Miller \\ University of Tennessee, Knoxville \\ Jacqueline C. Whittemore \\ University of Tennessee, Knoxville \\ Mee-Ja M. Sula \\ University of Tennessee, Knoxville
}

Follow this and additional works at: https://digitalcommons.murraystate.edu/faculty

\section{(c) (1) (9)}

This work is licensed under a Creative Commons Attribution-Noncommercial 4.0 License

\section{Recommended Citation}

Helgert, N. D., Miller, D. L., Whittemore, J. C., \& Sula, M.-J. M. (2021). Intrahistiocytic Storage of Clofazimine Crystals in a Cat. Veterinary Pathology, 58(2), 396-400. https://doi.org/10.1177/ 0300985820980717

This Peer Reviewed/Refereed Publication is brought to you for free and open access by Murray State's Digital Commons. It has been accepted for inclusion in Faculty \& Staff Research and Creative Activity by an authorized administrator of Murray State's Digital Commons. For more information, please contact msu.digitalcommons@murraystate.edu. 
1 Intrahistiocytic Storage of Clofazimine Crystals in a Cat

2 Nathan D. Helgert, Debra L. Miller, Jacqueline C. Whittemore and Mee-Ja M. Sula,

3 Breathitt Veterinary Center, Hutson School of Agriculture, Murray State University,

4 Hopkinsville, KY, USA (NH)

5 Department of Biomedical and Diagnostic Science, College of Veterinary Medicine,

6 University of Tennessee, Knoxville, TN, USA (DM,MS)

7 Department of Small Animal Clinical Science, College of Veterinary Medicine, University

8 of Tennessee, Knoxville, TN, USA (JW)

9 Corresponding author: N. Helgert. 101 MSU Dr. Hopkinsville, KY 42240. 270-881-3441,

10 nhelgert@murraystate.edu 
Abstract

2 A 13-year-old castrated male Maine coon cat with a 5-year history of atypical

3 mycobacteriosis was euthanized and submitted for necropsy. The cat had been kept in

4 clinical remission since diagnosis using a combination of the antimycobacterial drug

5 clofazimine and additional multimodal antimicrobial therapy. Grossly, tissues were

6 diffusely discolored red-brown to yellow. Histologically, the myocardial interstitum was

7 expanded by numerous, often multinucleated cells, which were distended by uniformly

8 shaped acicular cytoplasmic spaces. These cells were immunopositive for CD18 and

9 immunonegative for desmin, suggesting a histiocytic rather than muscular origin.

10 Macrophages in other tissues contained similar acicular spaces. Ultrastructurally, the

11 spaces were surrounded by two lipid membranes, resembling an autophagosome.

12 Based upon the clinical history and histologic, immunohistochemical, and ultrastructural

13 data, we diagnosed clofazimine crystal storage. To our knowledge, this is the first report

14 of clofazimine storage in a cat or within myocardial interstitial macrophages.

15 Keywords: clofazimine, feline, heart, histiocyte, mycobacteria, Mycobacterium avium

16 complex, mycobacteriosis 
Clofazimine is a highly lipophilic phenazine dye with antimycobacterial and anti-

2 inflammatory properties. This drug is listed as an essential medicine by the World

3 Health Organization and is most commonly utilized in humans for the treatment of

4 leprosy and multi-drug resistant tuberculosis. ${ }^{27}$ In humans, adverse effects are generally

5 self-limiting and include ichthyosis and pink discoloration of the skin in approximately

$694 \%$ of patients, ${ }^{20}$ less frequently, gastrointestinal pain ${ }^{20}$ and discoloration of the

7 sclera, ${ }^{3}$ and rarely clofazimine storage enteropathy. ${ }^{24,26}$ Histologically, clofazimine

8 storage enteropathy in humans is characterized by expansion of the gastrointestinal

9 lamina propria by crystal-laden macrophages following prolonged treatment with high doses of clofazimine. ${ }^{24,26}$ This condition can lead to unnecessary laparotomy, either due

11 to clinical signs suggestive of gastrointestinal obstruction ${ }^{24}$ or due to its radiologic

12 similarity to neoplastic processes. ${ }^{25}$ In humans, storage of clofazimine crystals has also

13 been reported in the macrophages of many tissues, including the lung, ${ }^{14,21,23}$ lymphoid 14 organs, ${ }^{19}$ liver, ${ }^{9}$ and eye. ${ }^{11}$

of mycobacteriosis. ${ }^{4,16,22}$ Adverse effects are rarely reported but include hepatotoxicity,

17 gastrointestinal signs, photosensitization, discoloration of the skin, and pitting corneal lesions ${ }^{4,16}$ Histologically confirmed cases of clofazimine storage enteropathy associated with treatment for mycobacteriosis have not been reported in domestic animals.

20 However, mice fed approximately $10 \mathrm{mg} / \mathrm{kg} /$ day of clofazimine developed pink

21 discoloration of the skin and hair and storage of clofazimine crystals within

22 macrophages in the intestine, liver, spleen, and lungs. ${ }^{2}$ Interestingly for the current 
1 transmission electron microscopy, intrahistiocytic clofazimine crystals have been shown

2 to be enveloped in a double lipid membrane, similar to those surrounding

3 autophagosomes. ${ }^{1}$

A 13-year-old castrated male Maine coon cat was submitted for necropsy. Eight

5 years prior to necropsy, the cat developed self-limiting lymphadenomegaly, followed by

6 immune-mediated retinal detachment, anemia, and thrombocytopenia. He was treated

7 with multiple immunosuppressive medications over the next three years, culminating in

8 hepatotoxicity due to cyclosporine overdosage 4.5 years prior to necropsy. Thereafter,

9 immunosuppression was gradually tapered and discontinued, and the cat developed

10 marked lymphadenomegaly and chemosis. Pyogranulomatous lymphadenitis with

11 intracytoplasmic negative-staining bacilli was identified following cytologic evaluation of

12 an enlarged superficial cervical lymph node. Mycobacterium avium was detected by

13 mycobacterial culture and PCRperformed on 4 excised enlarged retropharyngeal lymph

14 nodes. It was not possible to determine whether the cat's mycobacteriosis reflected

15 opportunistic infection secondary to chronic immunosuppression or if it had been the trigger for the cat's original presentation with immune-mediated retinal detachment, 17 anemia, and thrombocytopenia.

A detailed description of the clinical course of this case and the treatments are

19 presented in the Supplemental Materials. In short, for 8 years, the cat had been treated with various antimycobacterial agents and adjustments had been made as necessary to

21 maintain clinical remission. These medications included enrofloxacin (32.5mg, q24h),

22 rifampin (75mg, q24h), clarithromycin (62.5mg, q12h), amikacin (100mg, q24h),

23 ethambutol (300mg, q12h), moxifloxacin (30mg, q24h), minocycline (50mg, q24h), 
1 azithromycin (50mg, q24h), pradofloxacin (25mg, q24h), and clofazimine (50mg, q24h).

2 Clofazimine therapy was initiated 4 years prior to necropsy but was discontinued 5

3 months prior to euthanasia due to unavailability for use in veterinary medicine in the

$4 \quad$ United States. Three months following initiation of treatment with clofazimine, the cat's

5 skin, fur, and sclera developed a pink hue; this discoloration waned after discontinuation

6 of the medication. The cat was presented for euthanasia due to two weeks of weakness

7 and rapidly progressive weight loss. At the time of death, the cat was being treated with

8 azithromycin, pradofloxacin, and minocycline.

Gross findings at necropsy included purple-brown to red discoloration of most tissues, including skeletal muscle, kidney, liver, and bone marrow; adipose tissue was discolored yellow to brown. Tissues stained cutting surfaces bright pink, discolored

12 fixation solutions red-orange, and stained histological processing equipment bright pink

13 to dark red. The heart was subjectively enlarged with thickened ventricular walls and weighed $33.8 \mathrm{~g}$, which was $0.78 \%$ of body weight (University of Tennessee internal

15 reference range $0.3-0.45 \%$ ) and the liver weighed $240 \mathrm{~g}$, which was $5.5 \%$ of body weight (University of Tennessee internal reference range 3-3.5\%). Cavitary effusions were not

17 present. With the exception of the discoloration and multiple chronic renal infarcts, all organs were grossly unremarkable.

Samples of all major tissues were collected and fixed in $10 \%$ buffered neutral formalin, processed routinely, and routinely stained with hematoxylin and eosin for light

21 microscopic examination. For desmin immunohistochemistry Biocare's Decloaker and

22 Reveal Buffer (Biocare, Pacheco, CA) was used for antigen retrieval, and sections were 23 treated with monoclonal mouse anti- desmin antibodies (Dako, Santa Clara, CA, 
catalogue \#M0760; 1:100 dilution, 30 minutes) For CD18 immunohistochemistry

Carezyme I: Trypsin Kit (Biocare, Pacheco, CA) was used for antigen retrieval, then endogenous peroxidase activity was blocked with $3 \% \mathrm{H}_{2} \mathrm{O}_{2}$ and monoclonal mouse antifeline CD18 antibody was applied (clone Fe3.9f2, Peter Moore, University of CaliforniaDavis, Davis, CA; 1:10 dilution, 30 minutes,) ${ }^{6,8}$ Diaminobenzidine tetrahydrochloride was utilized as chromogen with hematoxylin counterstain. .

For transmission electron microscopy, formalin-fixed samples of myocardium were washed in $0.1 \mathrm{M}$ sodium phosphate buffer, post-fixed in buffered $2 \%$ osmium tetroxide for 60 minutes, washed in water, and dehydrated in a graded ethanol series with final dehydration in propylene oxide. Samples then were embedded in Embed 812 and semi-thin (1000nm) and thin sections (100nm) were prepared on a Leica EM UC7 ultra-microtome and stained with UranyLess stain (Electron Microscopy Sciences, Hatfield, PA) followed by Reynolds lead citrate to increase the contrast. Sections were imaged in a Zeiss Libra $200 \mathrm{MC}$ operating at $200 \mathrm{kV}$.

The most striking histologic feature was expansion of approximately $80 \%$ of the myocardial interstitium by many cells with up to 20 nuclei and abundant pale eosinophilic cytoplasm distended by regularly shaped clear acicular spaces (Figures 1 and 2). Similar cells, often multinucleated, were also identified within the interstitium of the skeletal muscle, although in lower numbers. These cells were immunoreactive for CD18 (Figure 3) and did not label with desmin, indicating a leukocytic origin. Given their multinucleation and similarity to Kupffer cells and pulmonary alveolar macropahges, they were interpreted as histiocytes. Additionally, myocardiocytes were variably sized (up to three-fold variation) and contained perinuclear brown pigment granules 
1 (lipofuscin). Kupffer cells, (Figure 4) and pulmonary alveolar macrophages also

2 contained similar clear, acicular spaces. Kupffer cells also contained abundant brown

3 granular pigment, which stained blue with Prussian blue (data not shown) and was

4 interpreted as hemosiderin. Notably, there was no evidence of these acicular spaces

5 within macrophages in the intestinal lamina propria.

Ultrastructurally, the spaces in myocardial macrophages were polygonal, electron-lucent, and lined by lipid membranes (Figures 5 and 6), consistent with an

8 autophagosome. Other histologic findings included regionally extensive acute hepatic necrosis and chronic tubulointerstitial nephritis with chronic infarcts. Staining of multiple tissues with Ziehl-Neelsen and Fite-Faraco acid fast stains did not demonstrate acidfast bacteria. Although considered a significant contributor to clinical decline, a definitive cause of the hepatic necrosis was not identified.

Given the historical treatment with clofazimine and the histologic and ultrastructural appearance of the crystalline spaces in macrophages, a diagnosis of clofazimine storage was made. Clofazimine crystals are soluble in organic solvents and alcohols and are therefore lost during routine tissue processing for histology and

17 ultrastructural study, leaving only clear acicular spaces. ${ }^{25}$ In order to see crystals histologically, frozen sections must be examined. Frozen samples were not collected in 19 this case.

To our knowledge, this is the first report of clofazimine accumulation within the

21 heart of a cat. Cardiotoxicity resulting in arrhythmias has previously been reported in

22 humans treated with clofazimine for prolonged periods 7,10 and cardiac accumulation of 23 clofazimine has been demonstrated in humans ${ }^{18}$ and rats. ${ }^{17}$ However, those studies did 
1 not include histologic evaluation for comparison to this case. In mice and humans with

2 many forms of chronic heart disease, the number of macrophages within the myocardial

3 interstitium increases. ${ }^{12}$ Cats with hypertrophic cardiomyopathy have recently been

4 shown to have increased numbers of macrophages within the myocardial interstitium. ${ }^{15}$

5 Although lacking the classical finding of myocardial disarray, the variably-sized

6 myocardiocytes in this cat, the subjective cardiomegaly with ventricular thickening, and

7 increased cardiac weight ( $0.78 \%$ of body weight) are suggestive of, though not

8 diagnostic for, hypertrophic cardiomyopathy. Alternatively, the increased heart weight

9 may have been the result of infiltration of the myocardium by clofazimine-laden

macrophages. A similar phenomenon of myocardial histiocytosis has also been

11 demonstrated in humans and mice with chronic kidney disease ${ }^{5}$ but not, to our

12 knowledge, in cats. The accumulation of clofazimine crystals within Kupffer cells ${ }^{2}$ and

13 pulmonary alveolar macrophages ${ }^{14,21,23}$ has been previously documented in

14 experimental and human literature and closely mirrors this case. Interestingly in this

15 case, and in contrast to humans ${ }^{24,26}$ and experimental models ${ }^{2}$, macrophages in the

16 intestinal lamina propria did not contain clofazimine crystals. The accumulation of

17 crystal-laden macrophages within the skeletal muscle intersititum, to the authors'

18 knowledge has not been previously reported and may suggest an unusual distribution of

19 clofazimine crystal storage in cats.

Although clofazimine is a mainstay of treatment of mycobacteriosis in cats, ${ }^{16,22}$

21 adverse effects rarely have been reported. Previously reported adverse effects are

22 limited to gastrointestinal upset and photosensitization, both of which typically resolve

23 following cessation of the medication. This report demonstrates clofazimine crystal 
storage within myocardial and skeletal muscle interstitial macrophages, Kupffer cells,

2 and pulmonary alveolar macrophages. Myocardial accumulation of clofazimine in this

3 case could represent an adverse effect of clofazimine, or could potentially a be

4 consequence of underlying cardiac pathological changes. In this case, the multiple

5 adverse effects associated with administration of other medications may reflect an

6 individual or species-specific sensitivity to various medications. Unfortunately, the role

7 of clofazimine in the clinical decline in this case is not determined.

8 References

1. Baik J, Rosania GR. Macrophages sequester clofazimine in an intracellular liquid crystal-like supramolecular organization. PLoS One. 2012;7(10).

2. Baik J, Stringer KA, Mane G, Rosania GR. Multiscale distribution and bioaccumulation analysis of clofazimine reveals a massive immune systemmediated xenobiotic sequestration response. Antimicrobial Agents and Chemotherapy. 2013;57:1218-1230.

3. Barot RK, Viswanath V, Pattiwar MS, Torsekar RG. Crystalline deposition in the cornea and conjunctiva secondary to long-term clofazimine therapy in a leprosy patient. Indian J Ophthalmol. 2011;59:328-329.

4. Bennett SL. Photosensitisation induced by clofazimine in a cat. Australian Veterinary Journal 2007;85(9):375-380.

5. Bugyei-Twum A, Abadeh A, Thai K, et al. Suppression of NLRP3 inflammasome activation ameliorates chronic kidney disease-induced cardiac fibrosis and diastolic dysfunction. Sci Rep. 2016;6.

6. Busch MDM, Reilly CM, Luff JA, Moore PF. Feline pulmonary langerhans cell histiocytosis with multiorgan involvement. Vet Pathol. 2008;45(6):816-824.

7. Choudhri SH, Harris L, Butany JW, Keystone JS. Clofazimine induced cardiotoxicity-a case report. Lepr Rev. 1995;66(1):63-68.

8. Cunha NP da, Ghisleni G, Scarampella F, et al. Cytologic and immunocytochemical characterization of feline progressive histiocytosis. Vet Clin Path. 2014;43(3):428436. 
9. Desikan K, Ramanugam K, Ramu G, Balakrishnan S. Autopsy findings in a case of lepromatous leprosy treated with clofazimine. Lepr Rev. 1975;46(3):181-189.

10. Diacon AH, Dawson R, von Groote-Bidlingmaier $F$, et al. Bactericidal activity of pyrazinamide and clofazimine alone and in combinations with pretomanid and bedaquiline. Am J Respir Crit Care Med. 2015;191(8):943-953.

11. Font RL, Sobol W, Matoba A. Polychromatic corneal and cConjunctival crystals secondary to clofazimine therapy in a leper. Ophthalmology. 1989;96(3):311-315.

12. Frodermann V, Nahrendorf M. Macrophages and cardiovascular health. Physiological Reviews. 2018;98(4):2523-2569.

13. Gunn-Moore DA, McFarland SE, Schock A, et al. Mycobacterial disease in a population of 339 cats in Great Britain: II. Histopathology of 225 cases, and treatment and outcome of 184 cases. Journal of Feline Medicine and Surgery. 2011;13(12):945-952.

14. Harbeck RJ, Worthen GS, Lebo TD, Peloquin CA. Clofazimine crystals in the cytoplasm of pulmonary macrophages. Ann Pharmacother. 1999;33(2):250-250.

15. Kitz S, Fonfara S, Hahn S, Hetzel U, Kipar A. Feline hypertrophic cardiomyopathy: The consequence of cardiomyocyte-initiated and macrophage-driven remodeling processes? Vet Pathol. 2019;56(4):565-575.

16. Malik R, Smits B, Reppas G, Laprie C, O'Brien C, Fyfe J. Ulcerated and nonulcerated nontuberculous cutaneous mycobacterial granulomas in cats and dogs. Vet Derm. 2013;24(1):146-e33.

17. Mamidi NV, Rajasekhar A, Prabhakar MC, Krishna DR. Tissue distribution and deposition of clofazimine in rat following subchronic treatment with or without rifampicin. Arzneimittelforschung. 1995;45(9):1029-1031.

18. Mansfield RE. Tissue concentrations of clofazimine (B663) in man. Am J Trop Med Hyg. 1974;23(6):1116-1119.

19. McDougall AC, Horsfall WR, Hede JE, Chaplin AJ. Splenic infarction and tissue accumulation of crystals associated with the use of clofazimine (Lamprene; B663) in the treatment of pyoderma gangrenosum. British Journal of Derm. 1980;102(2):227230.

20. Murashov MD, LaLone V, Rzeczycki PM, et al. The physicochemical basis of clofazimine-induced skin pigmentation. Journal of Investigative Dermatology. 2018;138(3):697-703.

21. Sandler E, Ng V, Hadley K. Clofazimine crystals in alveolar macrophages from a patient with the acquired immunodeficiency syndrome. Archives of Pathol and Lab Medicine. 1992;116(5):541-543. 
22. Sieber-Ruckstuhl NS, Sessions JK, Sanchez S, Latimer KS, Greene CE. Long-term cure of disseminated Mycobacterium avium infection in a cat. Vet Record. 2007;160(4):131-132.

23. Silverman JF, Holter JF, Berns LA, Benning TL, Neill JSA. Negative images due to clofazimine crystals simulating mai infection in a bronchoalveolar lavage specimen. Diag Cytopath. 1993;9(5):534-539.

24. Singh H, Azad K, Kaur K. Clofazimine-induced enteropathy in a patient of leprosy. Indian J Pharmacol. 2013;45(2):197-198.

25. Sukpanichnant S, Hargrove NS, Kachintorn U, et al. Clofazimine-induced crystalstoring histiocytosis producing chronic abdominal pain in a leprosy patient. The Americna Journal of Surg Path. 2000;24(1):129.

26. Szeto W, Garcia-Buitrago MT, Abbo L, Rosenblatt JD, Moshiree B, Morris MI. Clofazimine enteropathy: A rare and underrecognized complication of mycobacterial therapy. Open Forum Infect Dis. 2016;3(3).

27. Yoon GS, Keswani RK, Sud S, et al. Clofazimine biocrystal accumulation in macrophages upregulates interleukin 1 receptor antagonist production to induce a systemic anti-inflammatory state. Antimicrobial Agents and Chemotherapy. 2016;60(6):3470-3479.

Acknowledgements

The authors would like to acknowledge the University of Tennessee Advanced Microscopy and Imaging Center for instrument use, as well as scientific, and technical assistance.

Figure Legend

Figures 1-4. Clofazimine storage, cat. Figure 1. Myocardium. Diffusely, the interstitium is expanded by cells (macrophages) with abundant pale eosinophilic cytoplasm and intracytoplasmic acicular spaces. (HE) Figure 2. Myocardium. Higher magnification of Figure 1. The cells within the interstitium have up to 20 nuclei and their cytoplasm is distended by abundant acicular spaces (arrows). (HE) Figure 3. Myocardium. Cells within the interstitial spaces are immunoreactive to CD18, indicating a leukocytic origin. Immunohistochemistry for CD18. Figure 4. Liver. Fixed macrophages including Kupffer cells contain intracytoplasmic crystalline spaces and abundant granular golden-brown pigment (hemosiderin). (HE)

Figures 5 and 6. Clofazimine storage, myocardium, cat Transmission electron microscopy. Figure 5. Ultrastructurally, macrophages contain intracytoplasmic membrane-bound acicular spaces. Figure 6. Higher magnification of Figure 5. An acicular space (asterisk) is bound by a lipid bilayer (arrows). 